\title{
Pre-treatment effects against the diclofenac-induced toxicity by the aqueous leaf extract of Madhuca longifolia on female Wistar albino rats for 10 and 15 days
}

\author{
Jerine Peter Simon ${ }^{a}$, Sibi Karthik Saravanan ${ }^{a}$, Jayashree Sathiyanarayanana, Radhakrishnan Vidya ${ }^{b}$, \\ Usha Kumaric, Sabina Evan Prince ${ }^{\mathrm{a}^{*}}$ \\ ${ }^{a}$ Department of Biomedical Sciences, School of Biosciences and Technology (SBST), Vellore Institute of Technology, Vellore, India \\ ${ }^{b} V T T$ School of Agricultural Innovations and Advanced Learning (VALAL) Vellore Institute of Technology, Vellore, India \\ 'Faculty of Medicine, AIMST University, Malaysia
}

Received 15th August 2020 / Accepted 10th November 2020

\begin{abstract}
Diclofenac is used to treat rheumatism disorders, which are associated with the damages of renal, gastric and hepatic organs. Diclofenac is a pharmaceutical drug that is known to induce toxicity on its overdosage and long-term usage. Madbuca longifolia is known to have antioxidant, anti-inflammatory and anti-ulcer activity. It is an evergreen tree that is reported to have many ethnomedicinal uses. The other properties of Madbuca longifolia include anti-diabetic, analgesic and anti-microbial activities. Our study aims to evaluate the pre-treatment activity against the diclofenac-induced toxicity by the Madbuca longifolia aqueous leaf extract in Wistar albino rats for 10 and 15 days. Rats were divided as Group-I: Normal control, Group-II: Diclofenac on the last two days, Group-III and group-IIIa: Diclofenac + Aqueous Leaf Extract of Madhuca longifolia, Group-IV and group-IVb: Diclofenac + Silymarin, Group -V and group-Va: Aqueous Madhuca longifolia leaf extract. After the sacrifice, the rats were studied for antioxidant assay, renal enzyme markers, liver enzyme markers, and histopathological analysis of the kidney, stomach, intestine, and liver. As a result, we could identify that Madhuca longifolia has reduced the toxic changes in rats caused by diclofenac.
\end{abstract}

Keywords: Aqueous Madhuca longifolia leaf extract, Wistar albino rats, renal toxicity, gastrotoxicity, hepatotoxicity

\section{INTRODUCTION}

Diclofenac (DF) is a non-steroidal antiinflammatory drug (NSAID) with a phenylacetic derivative. Typically used in treating acute pain and rheumatoid arthritis. Recent studies in humans show the effect of DF in immune mechanisms, in part of idiosyncratic toxicity and its association with chronic pain in ankylosing spondylitis (McGettigan \& Henry, 2013). DF on overdosage and long-term usage is known to cause adverse effects like gastrointestinal toxicity, direct drug intense toxicity, renal toxicity, hepatotoxicity, and cardiovascular diseases. The liver plays a key function in the metabolism of cholesterol and fatty acids that are negatively affected by DF intake (Lin et al., 2002). The liver also produces endogenous compounds like bilirubin, bile salts, taurocholate, and bile pigments and produces blood clotting factors, proteins and enzymes. It metabolizes and breaks down toxic substances. Which may lead to the

*Author for correspondence: Sabina Evan Prince, School of Biosciences and Technology, Vellore Institute of Technology, Vellore-632014, Tamilnadu, India. Email - eps674@gmail.com 
accumulation of toxins in the damaged liver. The liver helps to filter toxins out of the portal circulation (Aneja et al., 2013). The drugs that we use under medication play a vital role in causing injury to the liver cells, which leads to inflammation of these cells (Zimmerman, 1998).

Plants that protect the liver contain an assortment of chemical constituents like monoterpenes, phenols, lignans, coumarins, carotenoids, fundamental oil, natural acids, xanthenes, glycosides, flavonoids, lipids, and alkaloids (Bodakhe \& Ram, 2007). Madbuca longifolia, a family of Sapotaceae, is a folk medicinal plant. Tribal people commonly use this for the treatment of snakebite as an antidote in the southern part of Tamilnadu, India (KretzRommel \& Boelsterli, 1993). Their flowers are used as an analgesic, diuretic agent and tonic. Madhuca longifolia leaves are used in the treatment of Cushing's disease and chronic bronchitis (Gaikwad et al., 2009; Walallawita et al., 2016). Our study aims to evaluate the pre-treatment effect against the DF-induced hepato, renal and gastro toxicity by the aqueous Madhuca longifolia leaf extract in female Wistar albino rats for 10 and 15 days.

\section{MATERIALS AND METHODS}

\section{Preparation of plant extract and chemicals}

Fresh Madhuca longifolia leaves were collected and authenticated by Prof. Jayaraman, Director of the Institute of Herbal Botany, Plant Anatomy Research Centre, Chennai, Tamil Nadu, India, in December 2016. The specimen authentication number is PARC/ 2016/3322. The samples were grinded with mortar and pestle. The sample was powdered and rinsed in distilled water. They were soaked and filtered with Whatman paper number 1. The sample aqueous leaf extract of Madbuca longifolia (ALEML) was dried in a water bath to get a yield of $10 \%(\mathrm{w} / \mathrm{w})$. Silymarin (SLY) tablet was obtained from Micro Laboratory Pvt, Ltd, Solan, Himachal Pradesh, India, which was administered through dissolving in sterile water. Injection vial was purchased from Unique Pharmaceutical Labs. Pvt. Ltd., Mumbai, Maharashtra, India.

\section{Animal house conditioning}

For the experiment, female Wistar albino rats of 160-190 $\mathrm{g}$ were obtained from the VIT Animal House, Vellore, Tamil Nadu, India. The intuitional animal ethical commission, VIT, Vellore, has authenticated the experiment on animals. The animal ethical clearance number is VIT / IAEC/13 / Feb13/21 that was obtained in accordance with Indian CPCSEA regulation. Animals were provided with the required environment.

\section{Animal experimental design}

The Female Wistar Albino rats were divided into groups of 6 rats each.

Group-I was the normal control group;

Group-II DF was induced with DF on the last two days $(50 \mathrm{mg} / \mathrm{kg}$ b.w. / day, i.p.),

Group-III was administrated with the pretreatment of aqueous Madhuca longifolia leaves extract $(500 \mathrm{mg} / \mathrm{kg}$ b.w. /day, p.o.) for 10 days in which DF was induced on 9th and 10th day,

Group-IIIa was administrated with the pretreatment of aqueous Madhuca longifolia leaves extract (500 mg/kg b.w. / day, p.o.) for all 15 days, and DF was induced on 14th and 15th day,

Group-IV was administrated with Silymarin $(25 \mathrm{mg} / \mathrm{kg}$ b.w./day, p.o.) as standard drug for 10 days, which was induced by DF on 9th and 10th day,

Group-IVa was administrated with the standard drug for 15 days, which was induced by DF on 14 th and 15 th day,

Group-V was administrated with aqueous Madbuca longifolia leaves extract $(500 \mathrm{mg} / \mathrm{kg}$ b.w. /day, p.o.) for 10 days,

Group-Va was administrated with aqueous Madhuca longifolia leaves extract $(500 \mathrm{mg} / \mathrm{kg}$ b.w. /day, p.o.) alone for 15 days.

Animals were allowed to fast for $12 \mathrm{~h}$ after the last experimental day, after the experimental period of 10 and 15 days. The animals were euthanized using anesthesia from the ether, and the blood was collected by heart puncture. An antioxidant and histopathological analysis was performed on the kidney, stomach, intestine, and liver tissue. The serum was collected by having the blood centrifuged for $10 \mathrm{~min}$ at $2000 \mathrm{rpm}$. 


\section{Biochemical and antioxidant analysis of the tissues}

Some portions of the tissue of the kidney, stomach, intestine and liver were homogenized and used to examine antioxidant properties such as superoxide dismutase (SOD) (Sarumathi et al., 2014), catalase (CAT) (Marklund \& Marklund, 1974) and reduced glutathione (GSH) (Sinha, 1972). Serum urea, uric acid and creatinine were measured using diagnostic kits obtained from Coral diagnostic Ltd, Chennai, Tamil Nadu, India. Hepatic enzyme markers such as aspartate aminotransferase (AST), alkaline phosphatase, alanine aminotransferase (ALT), total HDL cholesterol, triglyceride, albumin, total protein, total bilirubin, and direct bilirubin were used to detect the effect of ALEML on the liver tissue. Liver enzyme marker kits were purchased from Coral Diagnostic Ltd, Chennai, Tamil Nadu, India.

\section{Histological evaluation}

The samples were rinsed with $0.1 \mathrm{M}$ ice-cold phosphate-buffered saline (PBS) to remove blood from the kidney, stomach, intestine, and liver tissue. The sample was fixed in 10\% formalin for histopathology analysis. The sample was then mounted on a glass slide after deparaffinized and haematoxylin and eosin (H\&E) staining. It was visualized through the microscope for histopathological morphology analysis.

\section{Statistical analysis}

The findings obtained were expressed as a mean and standard deviation $( \pm)$ by using one way ANOVA, followed by the study by Student Newman-Keul. The values were considered $\mathrm{P}<$ 0.05 to be significant.

\section{RESULTS}

\section{Antioxidant efficiency of Madhuca longifolia in DF-induced rat}

The value of SOD, CAT and GSH of a kidney (Figure 1), stomach (Figure 2), intestine (Figure 3) and liver (Figure 4) are shown in pictorial representation respectively. The antioxidants released by the kidney have been reduced showing lower SOD, Catalase and GSH values than the control group in DF-injury. This case is similar in other organs like the stomach, intestine and liver. The rats treated with ALEML for 10 days and 15 days were observed to minimize the changes by DF that is similar to the rats treated with SLY, both on 10 days and 15 days study showed similar results on ALEML.

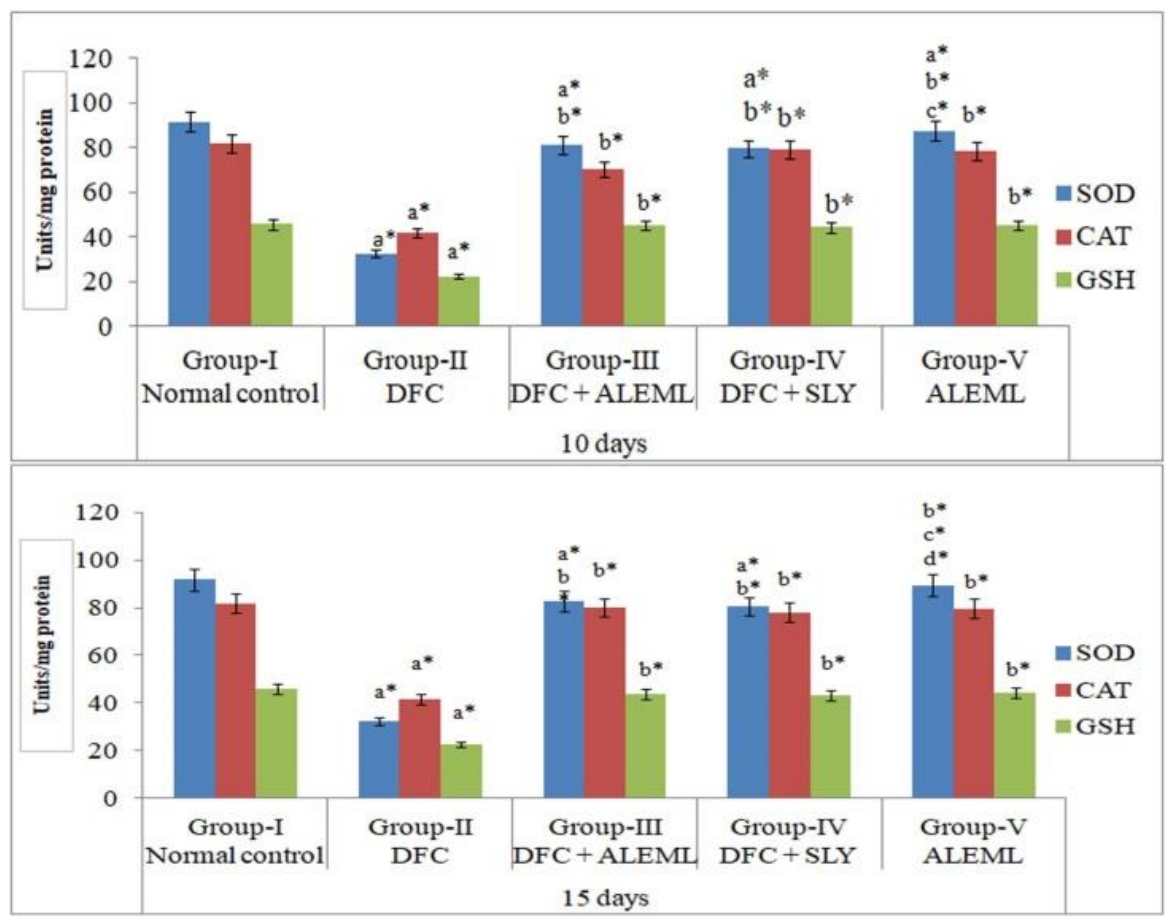

Figure 1.

Antioxidant efficiency of Madbuca longifolia on renal of DF-induced rats.

Every value is the mean SD of six rats. Comparisons were made as follows: a-Group-I versus GroupII, III, IIIa, IV, IVa, V, Va; bGroup-II versus Group-III, IIIa, IV, IVa, V, Va; c-Group-III versus Group-IV, IVa, V, Va; d-GroupIII versus Group-IV, IVa, V, Va; e-Group-IV versus Group-V, Va; f-Group-IV versus Group-V, Va; g-Group-V versus Group-Va.

The symbols $*_{\mathrm{p}}<0.05$ represent statistical significance. The statistical analysis was measured via one-way ANOVA, followed by the test of Student Newman-Keul. 

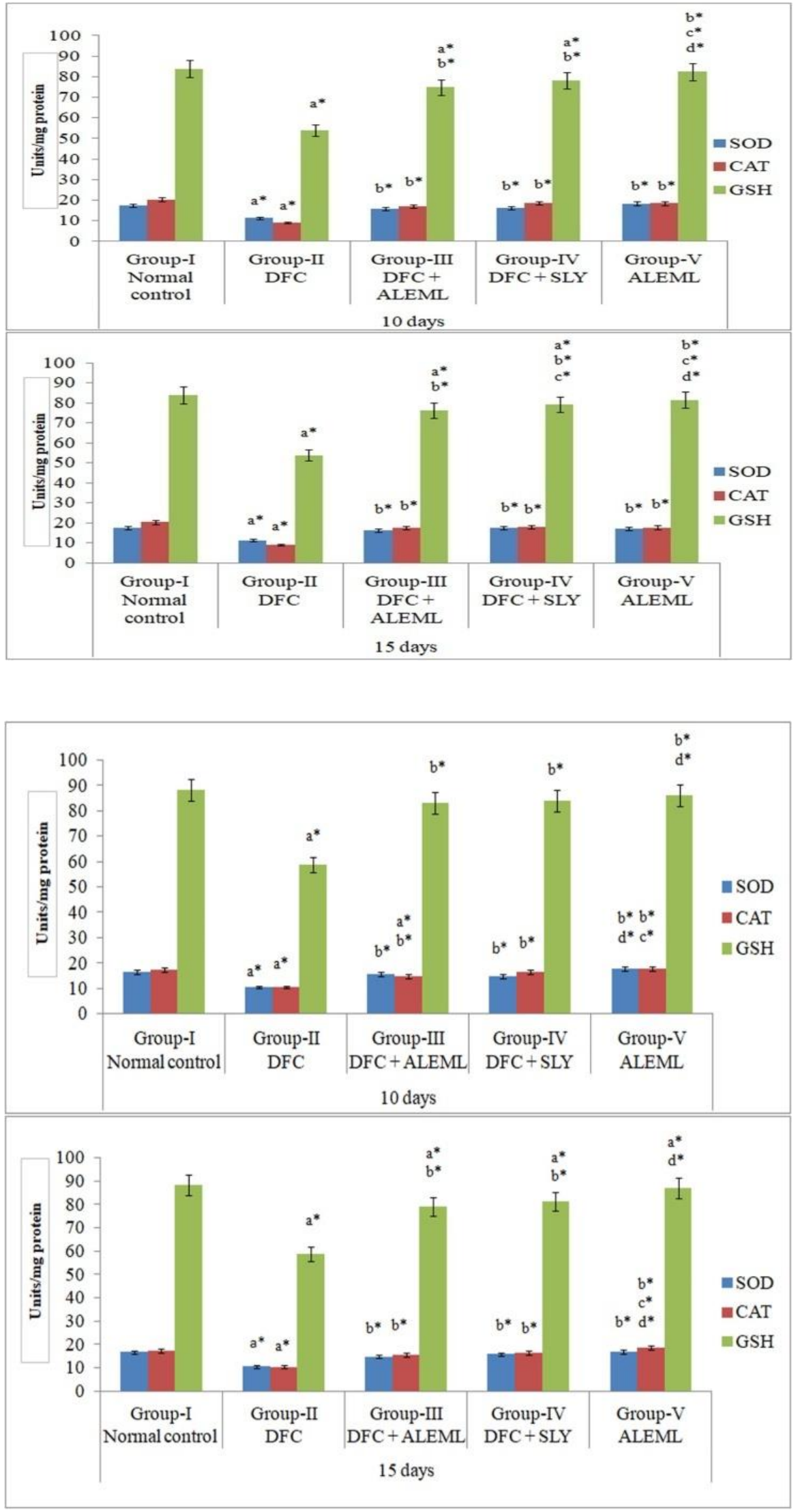

Figure 2.

Antioxidant efficiency of Madhuca longifolia on the stomach of DF-induced rats.

Every value is the mean SD of six rats. Comparisons were made as follows: a-Group-I versus GroupII, III, IIIa, IV, IVa, V, Va; bGroup-II versus Group-III, IIIa, IV, IVa, V, Va; c-Group-III versus Group-IV, IVa, V, Va; d-GroupIII versus Group-IV, IVa, V, Va; e-Group-IV versus Group-V, Va; f-Group-IV versus Group-V, Va; g-Group-V versus Group-Va. The symbols ${ }^{*} \mathrm{p}<0.05$ represent statistical significance. The statistical analysis was measured via one-way ANOVA, followed by the test of Student Newman-Keul.

\section{Figure 3.}

Antioxidant efficiency of Madhuca longifolia on the intestine of DF-induced rats.

Every value is the mean SD of six rats. Comparisons were made as follows: a-Group-I versus GroupII, III, IIIa, IV, IVa, V, Va; bGroup-II versus Group-III, IIIa, IV, IVa, V, Va; c-Group-III versus Group-IV, IVa, V, Va; d-GroupIII versus Group-IV, IVa, V, Va; e-Group-IV versus Group-V, Va; f-Group-IV versus Group-V, Va; g-Group-V versus Group-Va.

The symbols $*_{\mathrm{p}}<0.05$ represent statistical significance. The statistical analysis was measured via one-way ANOVA, followed by the test of Student Newman-Keul. 


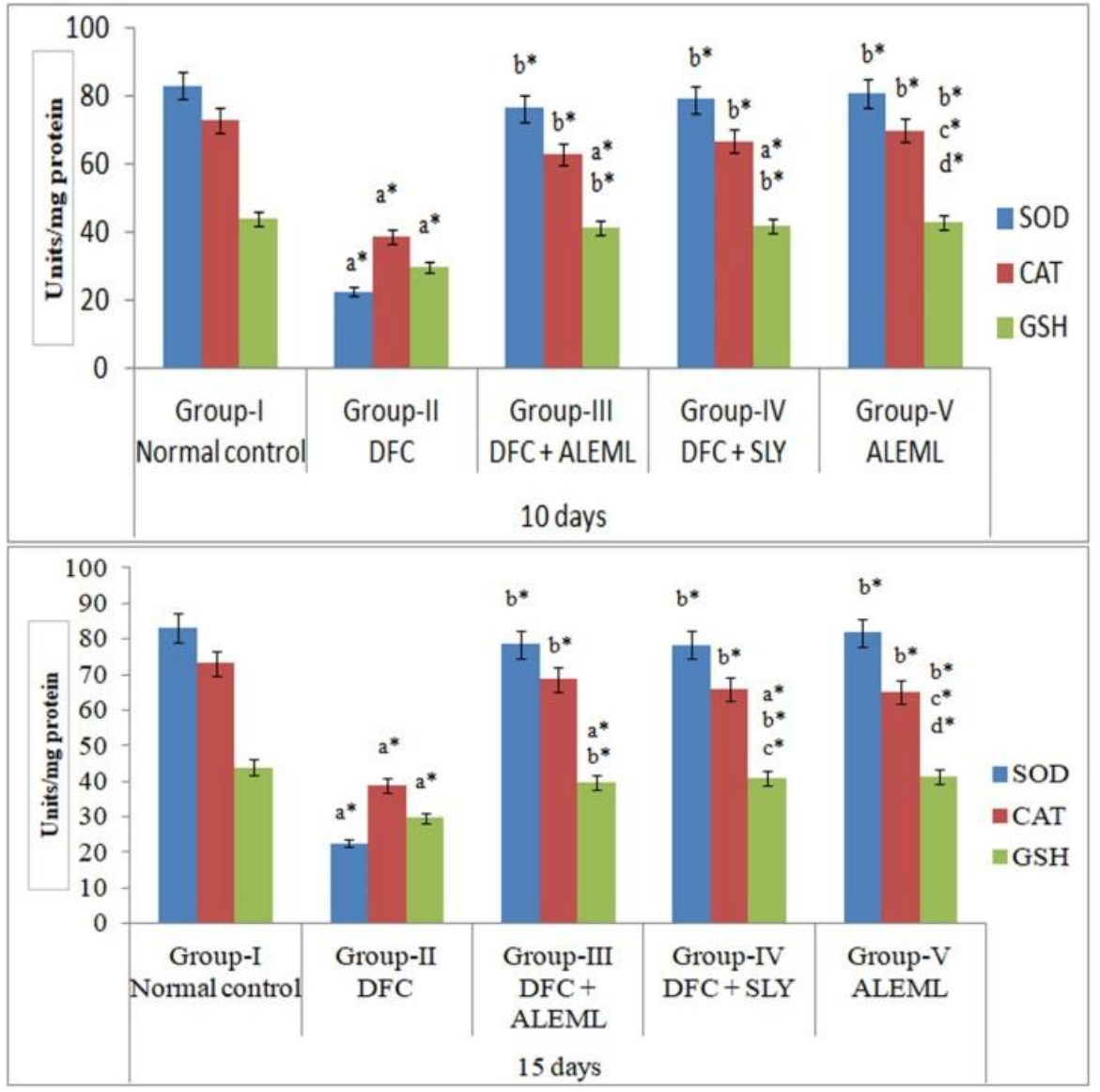

Figure 4.

Antioxidant efficiency of Madhuca longifolia on the liver of DF-induced rats.

Every value is the mean $\mathrm{SD}$ of six rats. Comparisons were made as follows: a-Group-I versus GroupII, III, IIIa, IV, IVa, V, Va; bGroup-II versus Group-III, IIIa, IV, IVa, V, Va; c-Group-III versus Group-IV, IVa, V, Va; dGroup-III versus Group-IV, IVa, V, Va; e-Group-IV versus GroupV, Va; f-Group-IV versus Group$\mathrm{V}, \mathrm{Va}$; g-Group-V versus GroupVa.

The symbols $*_{\mathrm{p}}<0.05$ represent statistical significance. The statistical analysis was measured via one-way ANOVA, followed by the test of Student NewmanKeul.

\section{Nephroprotective efficient of Madhuca longifolia in DF-treated rats}

Renal enzyme markers including urea, creatinine, and uric acid have been found to be notably higher than the normal control rats in Group-II DF rats. The levels of kidney markers were seen increased in multiple folds in rats induced with
DF alone which, when treated with ALEML for 10 days and 15 days, has shown the reduced release of urea, creatinine and uric acid in an execratory system that is similar to group-IV DF + SLY. The Group-V ALEML alone rats showed normalized levels of renal enzyme markers (Table 1).

Table 1. Efficient of renal markers on Madhuca longifolia treated DF-toxicity rats.

\begin{tabular}{llll}
\hline GROUP & Urea $(\mathbf{m g} / \mathbf{d L})$ & Uric acid $(\mathbf{m g} / \mathbf{d L})$ & Creatinine $(\mathrm{mg} / \mathbf{d L})$ \\
\hline Group-I & $15.52 \pm 0.98$ & $5.73 \pm 0.40$ & $0.58 \pm 0.57$ \\
Group-II & $76.71 \pm 0.71^{\mathrm{a}^{*}}$ & $26.7 \pm 0.50^{\mathrm{a}^{*}}$ & $4.88 \pm 0.36^{\mathrm{a}^{*}}$ \\
Group-III & $18.24 \pm 1.08^{\mathrm{a}^{*} \mathrm{~b}^{*}}$ & $7.19 \pm 0.73^{3^{*} \mathrm{~b}^{*}}$ & $1.07 \pm 0.32^{\mathrm{b}^{*}}$ \\
Group-IIIa & $19.64 \pm 1.00^{\mathrm{a}^{*} \mathrm{~b}^{*}}$ & $5.81 \pm 0.47^{\mathrm{b}^{*}}$ & $0.76 \pm 0.41^{\mathrm{b}^{*}}$ \\
Group-IV & $13.78 \pm 0.95^{\mathrm{b}^{*} \mathrm{c}^{*} \mathrm{~d}^{*}}$ & $5.97 \pm 0.50^{\mathrm{b}^{*}}$ & $0.90 \pm 0.46^{\mathrm{b}^{*}}$ \\
Group-Iva & $20.49 \pm 0.81^{\mathrm{a}^{*} \mathrm{~b}^{*} \mathrm{e}^{*}}$ & $5.58 \pm 0.40^{\mathrm{b}^{*}}$ & $0.90 \pm 0.28^{\mathrm{b}^{*}}$ \\
Group-V & $19.36 \pm 0.98^{\mathrm{a}^{*} \mathrm{~b}^{*} \mathrm{e}^{*}}$ & $6.07 \pm 0.59^{\mathrm{b}^{*}}$ & $1.04 \pm 0.54^{\mathrm{b}^{*}}$ \\
Group-Va & $25.54 \pm 0.86^{\mathrm{a}^{*} \mathrm{~b}^{*}}$ & $6.14 \pm 0.42^{\mathrm{b}^{*}}$ & $1.11 \pm 0.30^{\mathrm{b}^{*}}$ \\
\hline
\end{tabular}

Every value is the mean SD of six rats. Comparisons were made as follows: a-Group-I versus Group-II, III, IIIa, IV, Iva, V, Va; b-Group-II versus Group-III, IIIa, IV, Iva, V, Va; c-Group-III versus Group-IV, Iva, V, Va; d-Group-III versus GroupIV, Iva, V, Va; e-Group-Iv versus Group-V, Va; f-Group-Iv versus Group-V, Va; g-Group-V versus Group-Va.

The symbols ${ }^{*}<0.05$ represent statistical significance. The statistical analysis was measured via one-way ANOVA, followed by the test of Student Newman-Keul. 


\section{Hepatoprotective efficiency of Madhuca longifolia in DF-treated rats}

The value of total cholesterol and HDL was found to be minimized in DF-administrated rats, whereas the level of triglyceride was elevated significantly (Table 2); this condition is due to dyslipidemia. Albumin and total protein were found to be reduced in DF-induced rats. ALEML has demonstrated a hepatoprotective activity by normalizing the changes caused in DF-induced rats, which were similar to a standard drug. ALEML alone treated rats showed a similar result as Group-I.
Rats treated with DF have shown significant elevation $(\mathrm{P}<0.05)$ levels of liver function markers including ALT, AST, ALP (Figure 5), total protein and albumin (Figure 6), and also in total bilirubin and direct bilirubin (Table 3). During the liver injury, the liver enzymes will be leaked out in the bloodstream, so the elevated liver enzymes were found in DF-induced rats, which confirm the liver injury in the DF-treated group. The oral administration of ALEML for 10 and 15 days was observed to normalize the liver enzyme markers level in DF-induced rats, which is similar to SLYtreatment.

Table 2. Efficient of T. Cholestrol, HDL and Triglyceride on Madbuca longifolia treated DF-toxicity rats.

\begin{tabular}{|c|c|c|c|}
\hline GROUP & T. Cholestrol (mg/dL) & HDL (mg/dL) & Triglyceride (mg/dL) \\
\hline Group-I & $98.04 \pm 157$ & $47.19 \pm 1.27$ & $111.54 \pm 0.75$ \\
\hline Group-II & $36.63 \pm 1.49^{a^{*}}$ & $26.49 \pm 1.47 \mathrm{a}^{\mathrm{a}^{*}}$ & $275.45 \pm 0.66^{\mathrm{a}^{*}}$ \\
\hline Group-III & $86.52 \pm 0.77^{a^{*} b^{*}}$ & $56.74 \pm 1.18^{\mathrm{a}^{* \mathrm{~b}^{*}}}$ & $161.83 \pm 0.84^{\mathrm{a}^{*} \mathrm{~b}^{*}}$ \\
\hline Group-IIIa & $101.84 \pm 1.56^{\mathrm{a}^{*} \mathrm{~b}^{*} \mathrm{c} *}$ & $52.36 \pm 0.62^{\mathrm{a}^{*} \mathrm{~b} * \mathrm{c}^{*}}$ & $177.03 \pm 0.78^{\mathrm{a}^{*} \mathrm{~b}^{*} \mathrm{c} *}$ \\
\hline Group-IV & $94.12 \pm 1.27^{\mathrm{a}^{*} \mathrm{~b}^{*} \mathrm{c}^{*} \mathrm{~d}^{*}}$ & $51.24 \pm 0.988^{\mathrm{a}^{*} \mathrm{~b}^{*} \mathrm{c}^{*}}$ & $166.46 \pm 1.16^{a^{*} b^{*} c^{*} d^{*}}$ \\
\hline Group-Iva & $92.22 \pm 1.03^{a^{*} b^{*} c^{*} d^{*} e^{*}}$ & $52.60 \pm 1.225^{\mathrm{a}^{*} \mathrm{~b}^{*} \mathrm{c}^{*}}$ & $151.34 \pm 0.86^{\mathrm{a}^{*} \mathrm{~b}^{*} \mathrm{c}^{*} \mathrm{~d}^{*} \mathrm{e}^{*}}$ \\
\hline Group-V & $118.56 \pm 1.23^{a^{*} b^{*} c^{*} d^{*} f^{*}}$ & $54.40 \pm 0.65^{\mathrm{a}^{*} \mathrm{~b}^{*} \mathrm{e}^{*}}$ & $120.90 \pm 0.711^{\mathrm{a}^{*} \mathrm{~b}^{*} \mathrm{c}^{*} \mathrm{~d}^{*} \mathrm{f}}$ \\
\hline Group-Va & $127.83 \pm 1.05^{a^{*} b^{*} c^{*} d^{*} e^{*} f^{*} g^{*}}$ & $51.42 \pm 0.96^{\mathrm{a}^{*} \mathrm{~b}^{*} \mathrm{c}^{*} \mathrm{~g}^{*}}$ & $136.87 \pm 0.67^{\mathrm{a}^{*} \mathrm{~b}^{*} \mathrm{c}^{*} \mathrm{~d}^{*} \mathrm{e}^{*} \mathrm{f}^{*} \mathrm{~g}^{*}}$ \\
\hline
\end{tabular}

Every value is the mean SD of six rats. Comparisons were made as follows: a-Group-I versus Group-II, III, IIIa, IV, Iva, V, Va; b-Group-II versus Group-III, IIIa, IV, Iva, V, Va; c-Group-III versus Group-IV, Iva, V, Va; d-Group-III versus GroupIV, Iva, V, Va; e-Group-Iv versus Group-V, Va; f-Group-Iv versus Group-V, Va; g-Group-V versus Group-Va.

The symbols ${ }^{*} \mathrm{p}<0.05$ represent statistical significance. The statistical analysis was measured via one-way ANOVA, followed by the test of Student Newman-Keul.

Table 3. Effect of T. Bilirubin and D. Bilirubin on Madhuca longifolia treated DF-toxicity rats.

\begin{tabular}{lll}
\hline GROUP & T. Bilirubin $(\mathrm{mg} / \mathrm{dL})$ & D. Bilirubin $(\mathrm{mg} / \mathrm{dL})$ \\
\hline Group-I & $0.81 \pm 0.22$ & $0.06 \pm 0.01$ \\
Group-II & $3.58 \pm 0.36^{\mathrm{a}^{*}}$ & $3.17 \pm 0.18^{\mathrm{a}^{*}}$ \\
Group-III & $0.58 \pm 0.13^{\mathrm{b}^{*}}$ & $0.11 \pm 0.01^{\mathrm{b}^{*}}$ \\
Group-IIIa & $0.63 \pm 0.20^{\mathrm{b}^{*}}$ & $0.10 \pm 0.01^{\mathrm{b}^{*}}$ \\
Group-IV & $0.41 \pm 0.07^{\mathrm{b}^{*}}$ & $0.20 \pm 0.02^{\mathrm{b}^{*}}$ \\
Group-Iva & $0.80 \pm 0016^{\mathrm{b}^{*}}$ & $0.12 \pm 0.01^{\mathrm{b}^{*}}$ \\
Group-V & $0.70 \pm 0.06^{\mathrm{b}^{*}}$ & $0.14 \pm 0.01^{\mathrm{b}^{*}}$ \\
Group-Va & $0.86 \pm 0.19^{\mathrm{b}^{*}}$ & $0.14 \pm 0.02^{\mathrm{b}^{*}}$ \\
\hline
\end{tabular}

Every value is the mean SD of six rats. Comparisons were made as follows: a-Group-I versus Group-II, III, IIIa, IV, Iva, V, Va; b-Group-II versus Group-III, IIIa, IV, Iva, V, Va; c-Group-III versus Group-IV, Iva, V, Va; d-Group-III versus GroupIV, Iva, V, Va; e-Group-Iv versus Group-V, Va; f-Group-Iv versus Group-V, Va; g-Group-V versus Group-Va.

The symbols ${ }^{*} \mathrm{p}<0.05$ represent statistical significance. The statistical analysis was measured via one-way ANOVA, followed by the test of Student Newman-Keul. 


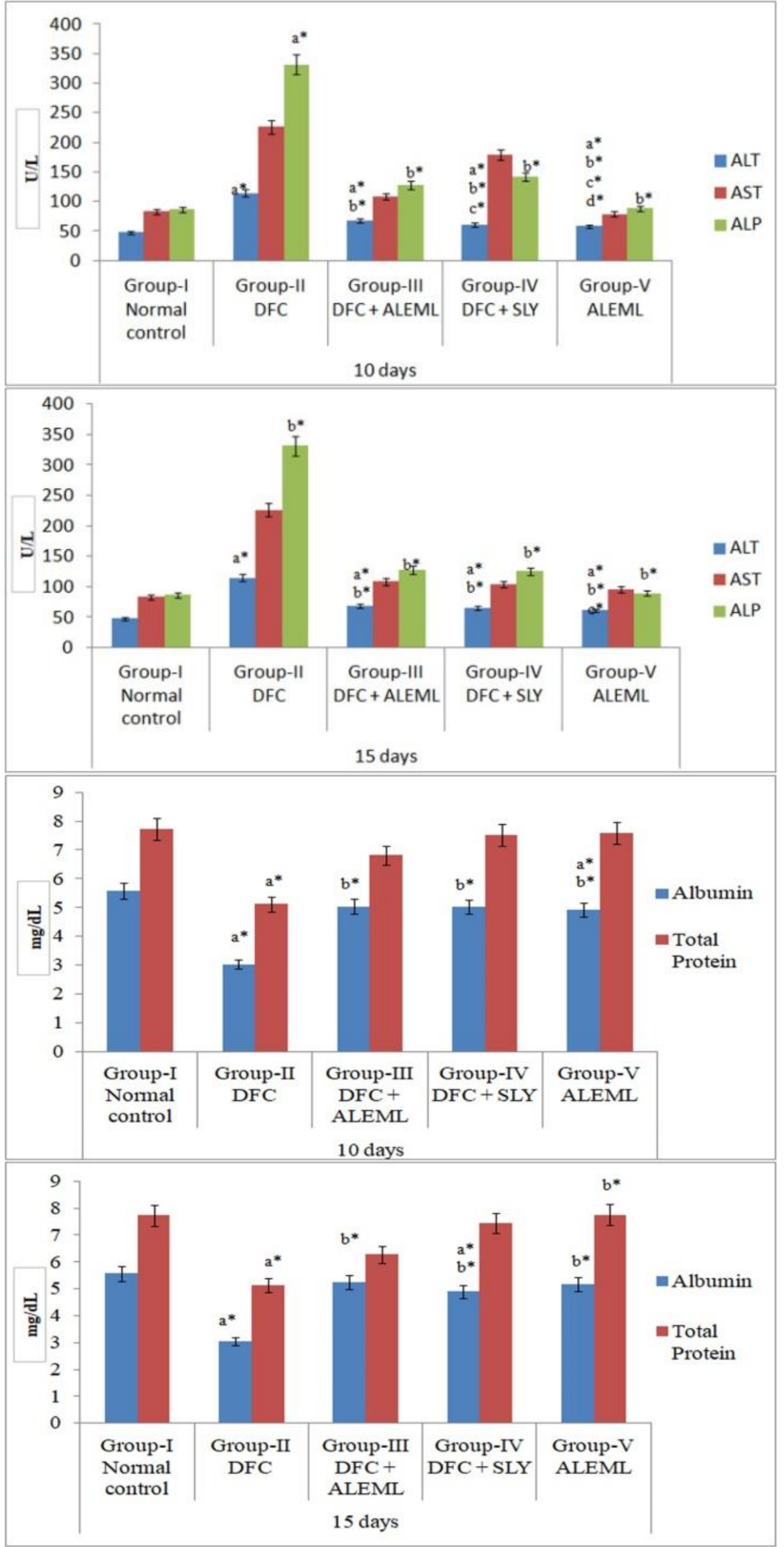

Figure 5.

Effect of ALT, AST and ALP on Madbuca longifolia treated DF-toxicity rats.

Every value is the mean SD of six rats. Comparisons were made as follows: a-Group-I versus GroupII, III, IIIa, IV, IVa, V, Va; bGroup-II versus Group-III, IIIa, IV, IVa, V, Va; c-Group-III versus Group-IV, IVa, V, Va; d-GroupIII versus Group-IV, IVa, V, Va; e-Group-IV versus Group-V, Va; f-Group-IV versus Group-V, Va; g-Group-V versus Group-Va.

The symbols ${ }^{*} \mathrm{p}<0.05$ represent statistical significance. The statistical analysis was measured via one-way ANOVA, followed by the test of Student Newman-Keul.

\section{Figure 6.}

Effect of albumin and total protein on Madhuca longifolia treated DF-toxicity rats.

Every value is the mean SD of six rats. Comparisons were made as follows: a-Group-I versus GroupII, III, IIIa, IV, IVa, V, Va; bGroup-II versus Group-III, IIIa, IV, IVa, V, Va; c-Group-III versus Group-IV, IVa, V, Va; d-GroupIII versus Group-IV, IVa, V, Va; e-Group-IV versus Group-V, Va; f-Group-IV versus Group-V, Va; g-Group-V versus Group-Va.

The symbols $* \mathrm{p}<0.05$ represent statistical significance. The statistical analysis was measured via one-way ANOVA, followed by the test of Student Newman-Keul. 


\section{Effect of Madhuca longifolia on histopathology of kidney, stomach, intestine and liver against DF-treated rats}

The histopathology of rat's kidney (Figure 7) from Group-II DF rats shows Peri-glomerular inflammation and tubular necrosis, which is due to the effect of DF in the rat's kidney. The GroupII DF rats also show Degeneration of their renal tubules. The Group-III DF + ALEML, GroupIIIa DF + ALEML, Group-IV DF + SLY, Group-Iva DF + SLY, Group-V ALEML alone, Group-Va ALEML alone rats were having a normal renal function like the Group-I rats, which is the control group. The histopathology of rat's stomach (Figure 8) from Group-II DF rats shows mucous erosion, inflammation and ulceration, which is due to the effect of DF in the rat's stomach. The Group-Iva DF + SLY rats show

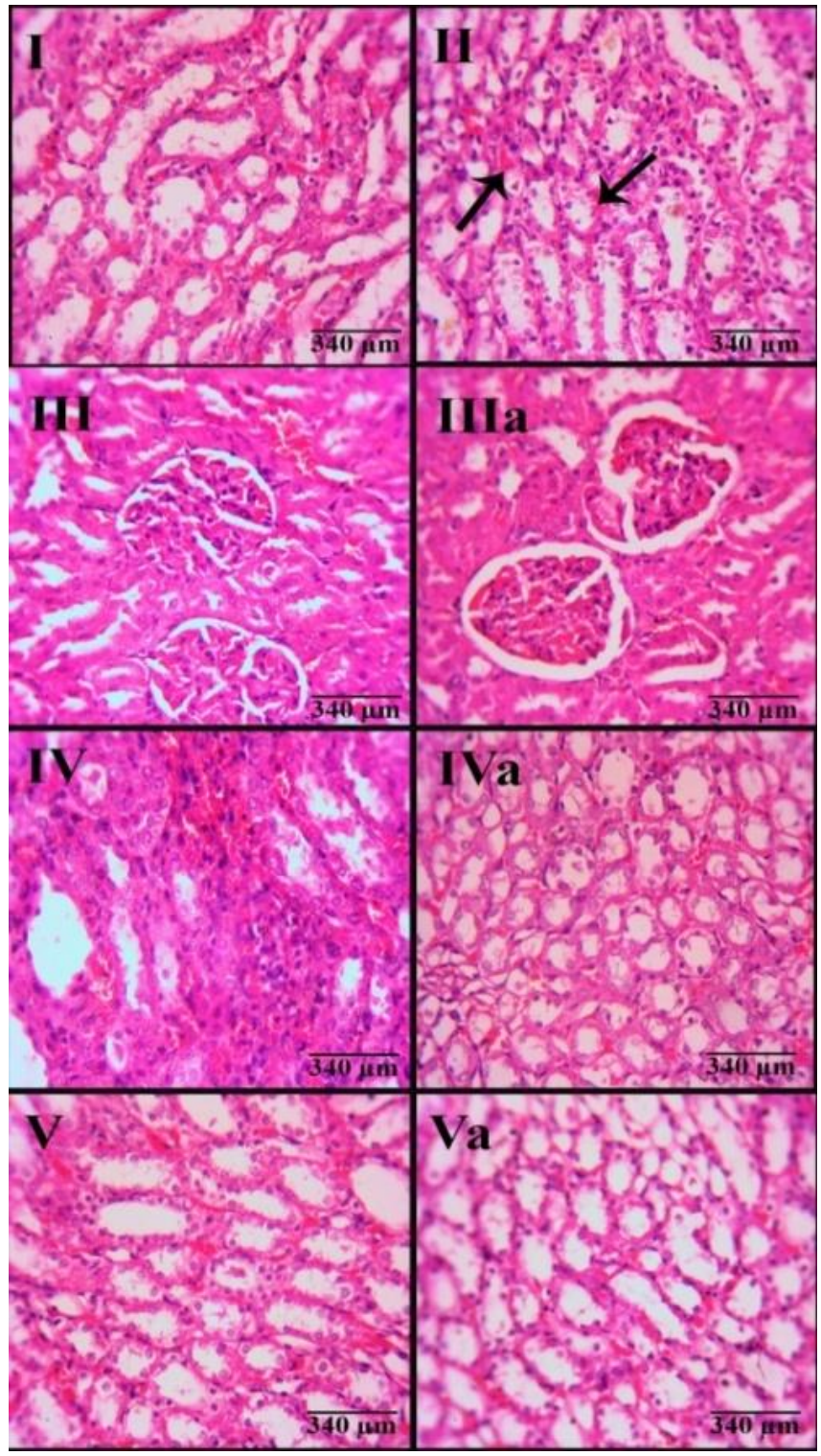

mild ulceration, which is due to SLY used for treating DF-injury. The remaining groups were at a normal level. The histopathology of rat's intestine (Figure 9) from Group-II DF rats shows severe ulceration, inflammation which is due to the effect of DF in the rat's intestine. The GroupIVa DF + SLY rats show moderate ulceration, which is due to SLY used for treating DF-injury. The histopathology of the liver (Figure 10) from Group-II DF rats shows Peri-portal inflammation, which is due to the effect of DF in the rat's liver. The Group-IIIa DF rats, which were treated with ALEML after DF-injury also showed moderate peri-portal inflammation while the Group-IVa DF + SLY rats, which were treated with SLY after DF-injury showed moderate periportal inflammation.

Figure 7. The nephroprotective effect of Madhuca longifolia on kidney histopathology of DF-induced rats.

H\&E staining: Group-I shows normal kidney histology with normal renal tubules; Group-II kidney tissue shows peri-glomerular inflammation and tubular necrosis; Group-III, Group-IIIa, Group-IV, Group-IVa, Group-V and Group-Va shows normal kidney histology with normal glomerular and renal tubules. 


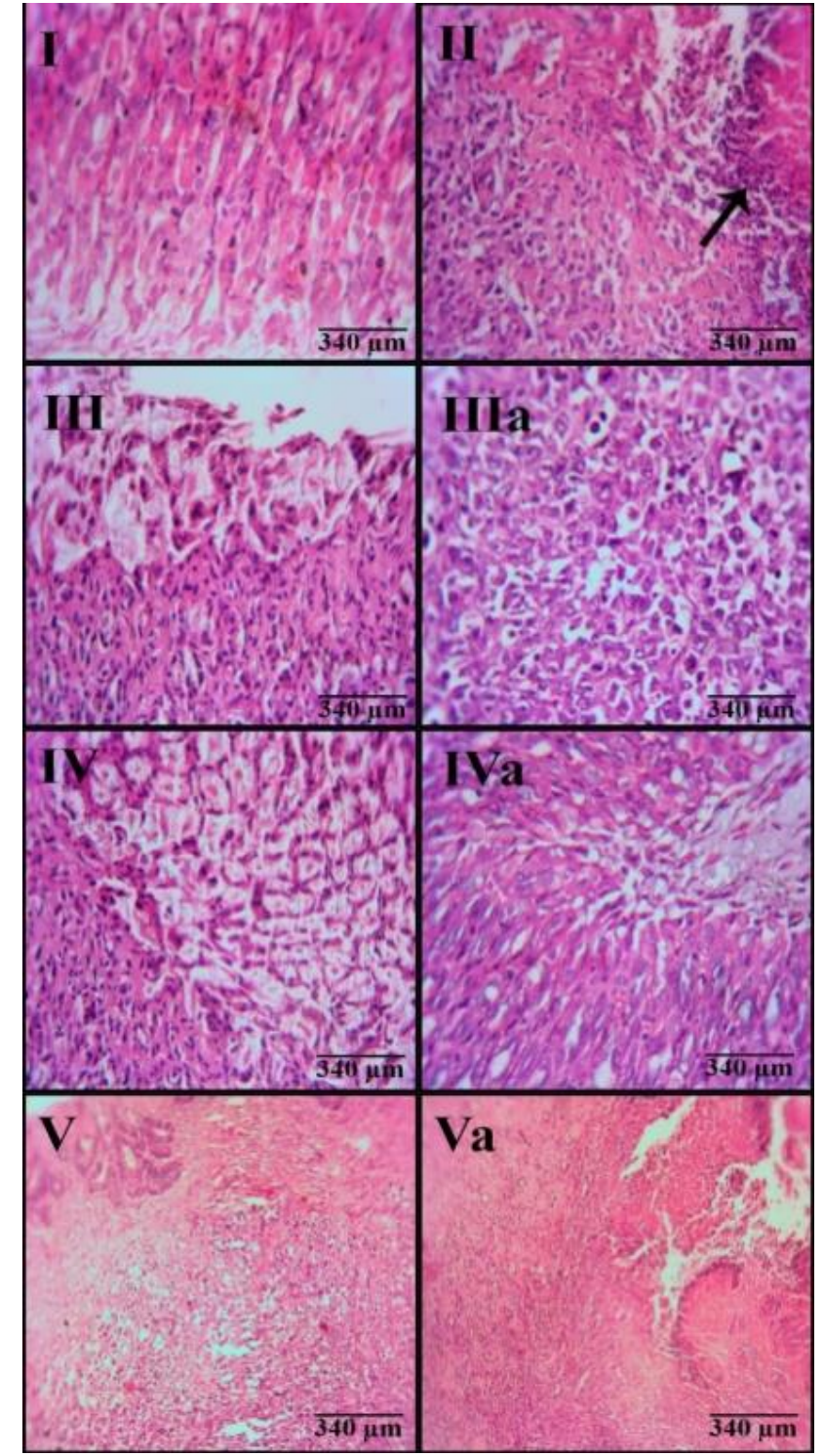

DISCUSSION

As we know, DF is a non-inflammatory drug that is used for pain and analgesics. It has been huge concern about the toxicity caused by DF that is hepato, renal and gastro toxicity. Its deposition and its bioactivation to intermediate reactive causes the production of oxidative stress, inflammation, tissue degeneration and necrosis suggested playing a role in organ damages (Adeyemi et al., 2019; Aycan et al., 2018). The histopathology change becomes predominant with DF treated rats has more tissue damage and inflammation with a dose of $50 \mathrm{mg} / \mathrm{kg}$. Since the hepatocytes receive blood flow, which has a
Figure 8. The gastro-protective effect of Madhuca longifolia on stomach histopathology of DFinduced rats.

H\&E staining: Group-I shows normal gastric mucosa; Group-II stomach tissue shows inflammation, mucous erosion and ulceration; Group-III, Group-IIIa, Group-IV, Group-IVa shows normal gastric mucosa; Group-IVa shows mild ulceration; Group-V, Group-Va shows normal gastric mucosa. reduced level of essential nutrients and oxygen that causes hepatic degeneration and periportal inflammation causes liver damage. The previous study elaborates the mechanism of DF hepatotoxicity in view of in vitro study shows the detoxified by GSH conjugation and toxic are moved out of the cell, where elimination of overdose DFis limited that lead to depletion of GSH and Whereby minimizing the cellular defense mechanism against oxidative stress that causes liver damage, along with this the generation of reactive metabolites, with high dosage the DF involve in mitochondrial injury, increase deposition of collagen fibers around portal tracts were found (Simon et al., 2019). 


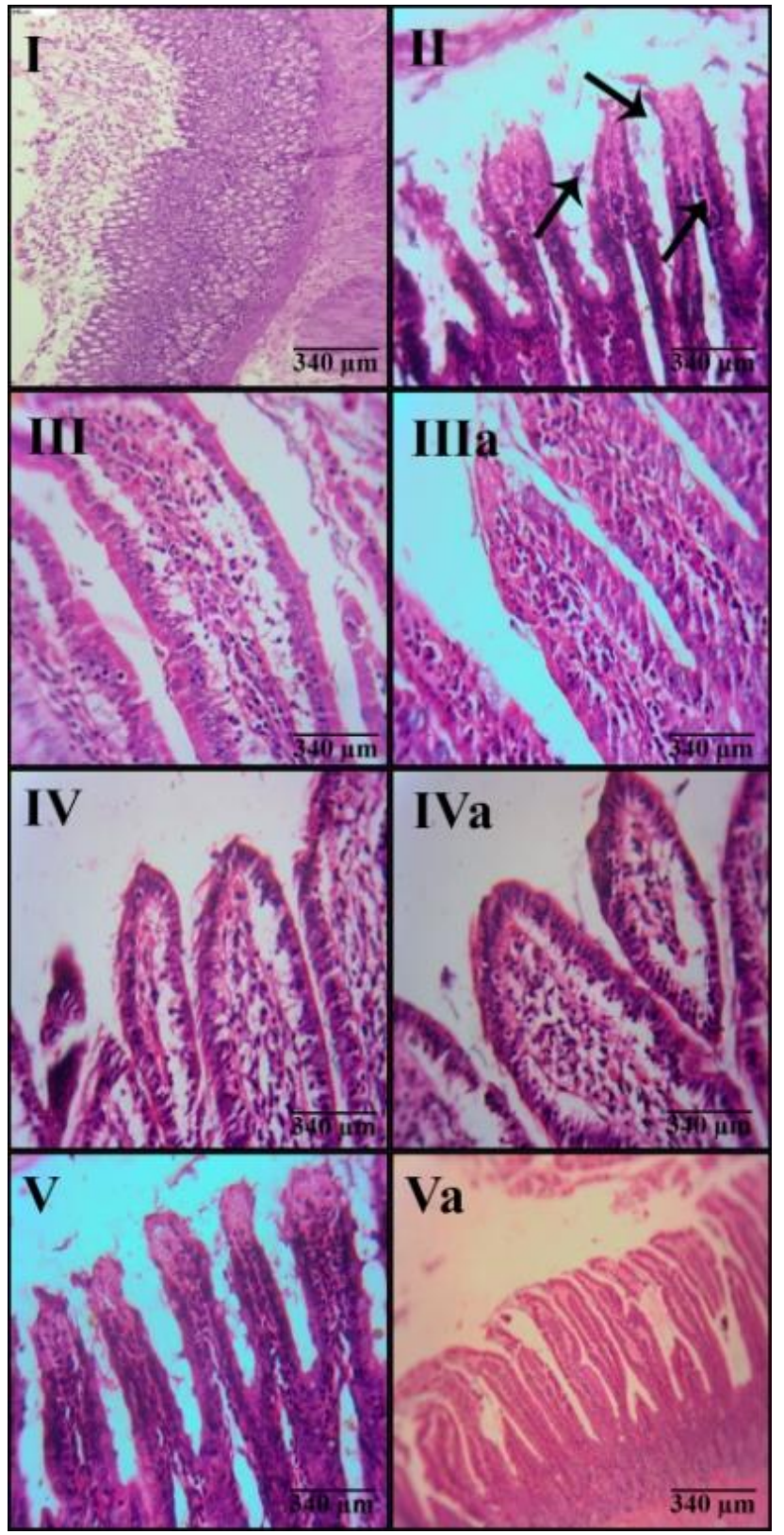

The levels of antioxidants in silymarin are decreased in the accumulation of hepatic collagen. The biochemical results of our study have shown that there is a significant elevation of AST, ALP and ALT on DF-induced rats. The rats treated with Madbuca longifolia has normalized the elevated levels. The acute and chronic liver injury was identified with an increase in serum concentration of AST and ALT.in The recent studies shows that the increase in AST and ALT level on the administration of DF is known to indicate subchronic hepatotoxicity along with severe damage in liver tissue, which also reduces insulin sensitivity, increases free fatty acid and triglycerides and decreased glucose tolerant.
Figure 9. The gastro-protective effect of Madhuca longifolia on intestine histopathology of DFinduced rats.

H\&E staining: Group-I shows normal gastric mucosa; Group-II intestine shows severe ulceration; Group-III, Group-IIIa, Group-IV, Group-IVa shows normal gastric mucosa; GroupIVa shows moderate ulceration; Group-V and Group-Va shows normal gastric mucosa.

Increase serum bilirubin protects from strokes. The decrease in albumin and total protein was observed in the present study, but it's already studied previously (Liu et al., 2018; Yamamura et al., 2019). The benefits of Madbuca longifolia in experimental studies concern hepatoprotective effects that were reported in the previous study (Kumar et al., 2018). Madhuca longifoia is reported to increase the antioxidant potential in the system and also help in the elevation of free radicals caused by oxidative stress. During chemical reactions or drug metabolism, the free radicals are elevated in the body and thus increase the oxidative stress in the body (Adeyemi et al., 2019). This increased oxidative stress is suppressed by 
the in-built antioxidant mechanism of the body. $\mathrm{DF}$ is reported to damage the mechanism of this antioxidant mechanism by depleting the GSH conjugation. In the current study, Madhuca longifolia was observed to restore the antioxidant mechanism (Jha \& Mazumder, 2018). This shows the potential of Madbuca longifolia in reducing the free radicals and thus normalizing the tissue histopathology. This mechanism of Madbuca longifolia is similar in treating hepatotoxicity, nephrotoxicity and gastrotoxicity (Mohod \& Bodhankar, 2013). It also showed the

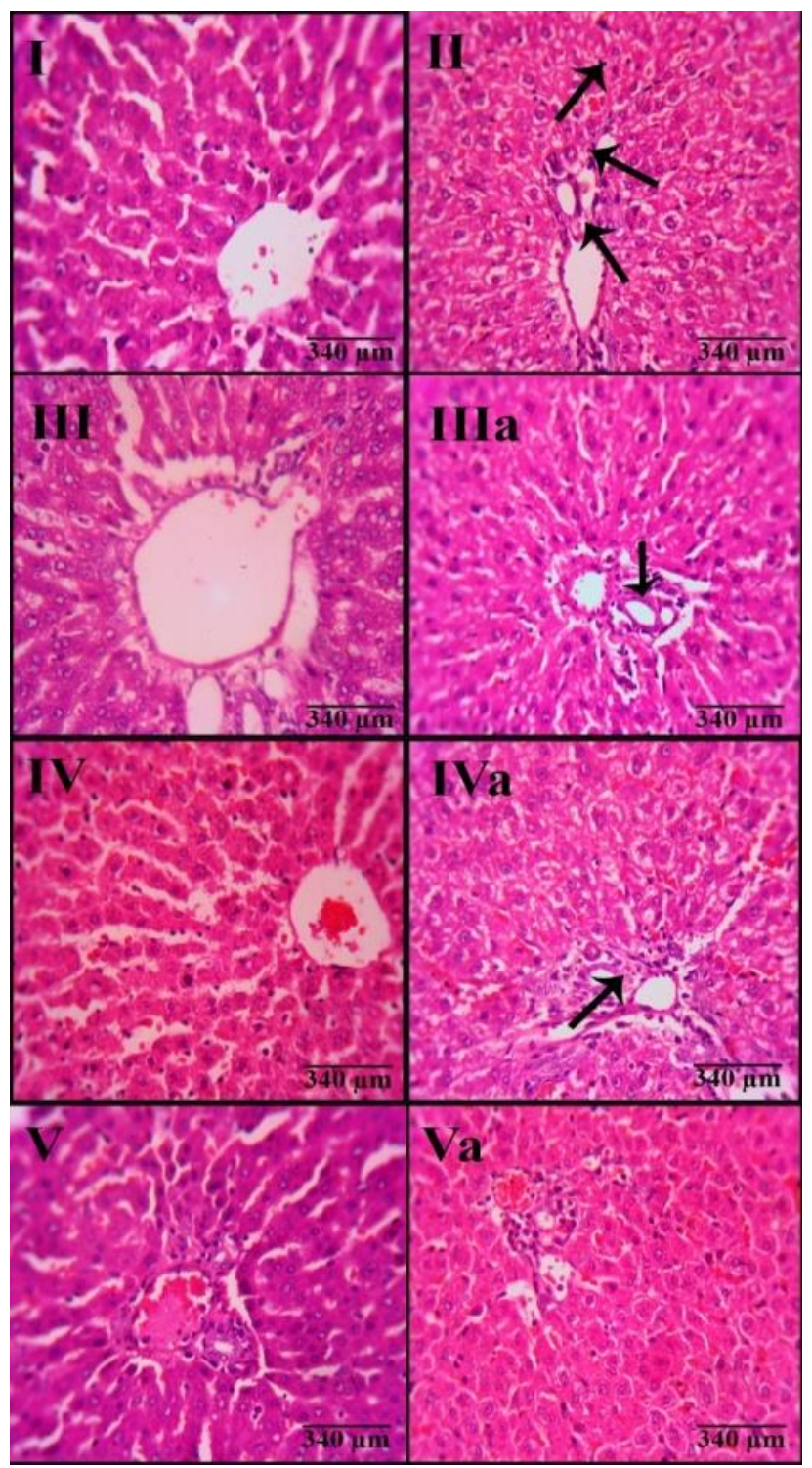

effectiveness of carbon tetrachloride-induced cellular and membrane damage in organs. The present study shows that hepato, gastro and renal damage are caused by DF was mild with its therapeutic dosage, administration of Madbuca longifolia intake will be a best alternative drug for silymarin and natural antioxidant agent considered to encourage its uses in future research work. This can be further studied by understanding the metabolic pathway of Madbuca longifolia and its active compounds in treating toxicity.

Figure 10. The hepatoprotective effect of Madhuca longifolia on liver histopathology of DF-induced rats.

H\&E staining: Group-I shows normal liver histology; Group-II liver tissue shows peri-portal inflammation and hepatic degeneration; Group-III shows normal liver histology and Group-IIIa shows moderate peri-portal inflammation, GroupIV shows normal liver morphology; Group-IV shows normal liver histology; Group-IVa shows hepatic degradation and mild peri-portal inflammation and mild periportal inflammation; Group-V and Group-Va show normal liver histology. 


\section{CONCLUSION}

The current study has shown the hepatoprotective, nephroprotective and gastroprotective activity of ALEML for 10 and 15 days against DF-induced rats through antioxidant assay, renal enzyme, liver enzyme markers and histopathology analysis. ALEML for 10 and 15 days has confirmed its potential activity as an antioxidant, anti-inflammatory and anti-ulcer agent.

\section{ACKNOWLEDGEMENTS}

The authors thank VIT for providing 'VIT SEED GRANT' for carrying out this research work.

\section{REFERENCES}

Adeyemi, W. J., Omoniyi, J. A., Olayiwola, A., Ibrahim, M., Ogunyemi, O., \& Olayaki, L. A. 2019. Elevated reproductive toxicity effects of diclofenac after withdrawal: Investigation of the therapeutic role of melatonin. Toxicology Reports 6: 571-577.

Aneja, S., Vats, M., Aggarwal, S., \& Sardana, S. 2013. Phytochemistry and hepatoprotective activity of aqueous extract of Amaranthus tricolor Linn. roots. Journal of Ayurveda and Integrative Medicine 4(4): 211-215.

Aycan, İ. Ö., Elpek, Ö., Akkaya, B., Kıraç, E., Tuzcu, H., Kaya, S., Coşkunfirat, N., \& Aslan, M. 2018. Diclofenac induced gastrointestinal and renal toxicity is alleviated by thymoquinone treatment. Food and Chemical Toxicology 118: 795-804.

Bodakhe, S. H. \& Ram, A. 2007. Hepatoprotective properties of Bauhinia variegata bark extract. Yakugaku Zasshi: Journal of the Pharmaceutical Society of Japan 127(9): 1503-1507.

Gaikwad, R. D., Ahmed, M. L., Khalid, M. S., \& Swamy, P. 2009. Anti-inflammatory activity of Madhuca longifolia seed saponin mixture. Pharmacentical Biology 47(7): 592-597.

Jha, D., \& Mazumder, P. M. 2018. Biological, chemical and pharmacological aspects of Madhuca longifolia. Asian Pacific Journal of Tropical Medicine 11(1): 9.

Kretz-Rommel, A. \& Boelsterli, U. A. 1993. Diclofenac covalent protein binding is dependent on acyl glucuronide formation and is inversely related to P450-mediated acute cell injury in cultured rat hepatocytes. Toxicology and Applied Pharmacology 120(1): 155-161.

Kumar, R., Bora, G. S., Banerjee, S., Mandal, A., \& Naiya, T. K. 2018. Application of naturally extracted surfactant from Madbuca longifolia to improve the flow properties of heavy crude oil through horizontal pipeline. Journal of Petroleum Science and Engineering 168: 178-189.

Lin, S.-C., Lin, C.-H., Lin, C.-C., Lin, Y.-H., Chen, C.-F., Chen, I.C., \& Wang, L.-Y. 2002. Hepatoprotective effects of
Arctium lappa linne on liver injuries induced by chronic ethanol consumption and potentiated by carbon tetrachloride. Journal of Biomedical Science 9(5): 401-409.

Liu, C.-F., Zhou, W.-N., Lu, Z., Wang, X.-T., \& Qiu, Z.-H. 2018. The associations between liver enzymes and the risk of metabolic syndrome in the elderly. Experimental Gerontology 106: 132-136.

Marklund, S. \& Marklund, G. 1974. Involvement of the superoxide anion radical in the autoxidation of pyrogallol and a convenient assay for superoxide dismutase. European Journal of Biochemistry 47(3): 469-474.

McGettigan, P., \& Henry, D. 2013. Use of non-steroidal antiinflammatory drugs that elevate cardiovascular risk: An examination of sales and essential medicines lists in low-, middle-, and high-income countries. PLOS Medicine 10(2): e1001388.

Mohod, S. M. \& Bodhankar, S. L. 2013. Antiulcer activity of aqueous extract of leaves of Madbuca indica J. F. Gmel against naproxen induced gastric mucosal injury in rats. Journal of Acute Disease 2(2): 127-133.

Sarumathi, A., Sethupathy, S., \& Saravanan, N. 2014. The protective efficacy of spirulina against bacterial endotoxin potentiated alcoholic liver disease. Journal of Functional Foods 9: 254-263.

Simon, J. P., Parthasarathy, M., Nithyanandham, S., Katturaja, R., Namachivayam, A., \& Prince, S. E. 2019. Protective effect of the ethanolic and methanolic leaf extracts of Madhuca longifolia against diclofenac-induced toxicity in female Wistar albino rats. Pharmacological Reports.

Sinha, A. K. 1972. Colorimetric assay of catalase. Analytical Biochemistry 47(2): 389-394.

Walallawita, W. K. U. S., Bopitiya, D., Sivakanthan, S., Jayawardana, N. W. I. A., \& Madhujith, T. 2016. Comparison of oxidative stability of sesame (Sesamum indicum), soybean (Glycine max) and mahua (Mee) (Madhuca longifolia) oils against photo-oxidation and autoxidation. Procedia Food Science, International Conference of Sabaragamuwa University of Sri Lanka 2015 (ICSUSL 2015) 6: 204-207.

Yamamura, K., Sakamoto, I., Morihana, E., Hirata, Y., Nagata, H., Yamasaki, Y., Okumura, Y., Kohashi, K., Koto, K., Tsutsui, H., \& Ohga, S. 2019. Elevated non-invasive liver fibrosis markers and risk of liver carcinoma in adult patients after repair of tetralogy of Fallot. International Journal of Cardiology 287: 121-126.

Zimmerman, H. J. 1998. Acetaminophen hepatotoxicity. Clinics in Liver Disease 2(3): 523-541. 